\title{
Photocrosslinkable starch-based polymers for ophthalmologic drug delivery
}

\author{
A.P. Vieira ${ }^{a}$, P. Ferreira ${ }^{a, b}$, J.F.J. Coelho ${ }^{c}$, M.H. Gil ${ }^{a}, *$ \\ a Chemical Engineering Department, University of Coimbra, Pólo II, Rua Sílvio Lima, 3030-790 Coimbra, Portugal \\ ${ }^{\mathrm{b}}$ Department of Health Sciences, Portuguese Catholic University, Estrada da Circunvalação, 3504-505 Viseu, Portugal

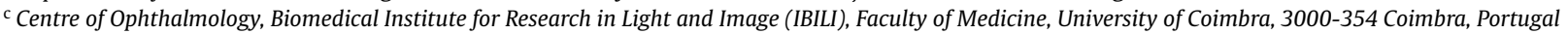

\section{A R T I C L E I N F O}

\section{Article history:}

Received 6 May 2008

Received in revised form 19 May 2008

Accepted 2 June 2008

Available online 6 June 2008

\section{Keywords:}

Starch

Drug delivery

Photocrosslinking

\begin{abstract}
A B S T R A C T
This study focused on the development and characterization of a starch-based polymer with urethane linkages to be used as a controlled drug delivery system for biomedical applications. Starch was modified with 2-isocyanatoethyl methacrylate in order to obtain a polymer containing carbon-carbon double bonds in its structure. This modified starch was then used to produce films by UV irradiation using Irgacure ${ }^{\circledR} 2959$ (CIBA) as the photoinitiator. The modified polymer was characterized by attenuated total reflectanceFourier transform infrared (ATR-FTIR) spectroscopy. The swelling capacity, in artificial lachrymal fluid (performed both at room temperature and physiological temperature), and water contact angles measurements were determined. The in vitro biodegradation in artificial lachrymal fluid supplemented with lysozyme was also studied. Scanning electronic microscopy (SEM) was used to characterize the morphology of the materials immediately after synthesis and after biodegradation. Timolol maleate and sodium flurbiprofen were immobilized by adsorption and their in vitro release profiles were followed spectroscopically.
\end{abstract}

(C) 2008 Elsevier B.V. All rights reserved.

\section{Introduction}

Conventional ocular therapy for the treatment of acute and chronic diseases makes use of topical appliance of eye drops. This type of therapeutics represents nearly $90 \%$ of the marketed formulations. Still, this kind of appliance has a limited efficacy that is due to several factors. One of the major limiting factors for drug absorption from the lachrymal fluid into the anterior chamber, after eye drop administration, is the low permeability of the corneal epithelium that results in a very low (around 5\%) drug absorption by the cornea [1]. The remaining amount of drug flows with tears through the upper and lower canalicully into the nasolachrymal ducts [2] and consequently may cause unwanted systemic side effects [3]. The self-protective mechanisms of the eye, such as rapid tear turnover, limit the absorption of the instilled drug in the eye [4]. In addition, application of ophthalmic drugs as drops results in a rapid variation in drug delivery rates to the cornea that limits the efficacy of therapeutic systems [5].

In order to improve the patient compliance for delivering the medications there is the need for finding some new implantable devices which could deliver the drugs in a long-lasting controlled

\footnotetext{
* Corresponding author at: Departamento de Engenharia Química, Universidade de Coimbra, Polo II, Pinhal de Marrocos, 3030-290 Coimbra, Portugal.

Tel.: +351 239798743; fax: +351 239798703.

E-mail address: hgil@eq.uc.pt (M.H. Gil).
}

manner [6]. Using this strategy, the drug loss associated with systemic absorption would be minimized, and the resident time of the drug in the tear film increased [7]. An alternative approach to optimize ophthalmic drug delivery is the adaptation of bioadhesive systems [8], namely mucoadhesive ones, which have been proved to be successful in oral applications [9].

Bioadhesive delivery systems are designed to adhere to various tissue surfaces, mainly the mucosal epithelium, and can provide a localized delivery of an active agent to a specific site in the body such as the eye [3]. Some authors have come to the conclusion that mucoadhesion requires an expanded and hydrated polymer network [10]. In fact, according to Mulhbacher et al. [11] it is possible to describe three factors involved in the process of bioadhesion. The first is related to wetting and the swelling of the polymer, while the second concerns to the interpenetration of the polymeric chains with mucin. Finally, the third is related to the establishment of weak physical bonds between mucin and polymeric chains. These last interactions can be ionic, Van der Waals or hydrogen bonds and although they are, as said before, weak interactions individually, they can associate in a final strong adhesion phenomenon. Considering this description, the most suitable polymers to be applied as mucoadhesive agents, will be hydrophilic macromolecules containing numerous hydrogen bond forming groups [12]. Polysaccharides, being naturally occurring biomolecules, seem suitable compounds to develop this kind of systems, since they are well know as highly hydrophilic as well as biodegradable and biocompatible. In addition, this kind of molecule can be modified to change its physical 
characteristics and, hence, improve its applicability for a specific application. Among these, starch is one of the most abundant available polymers obtained from natural products, and it was chosen to perform this study. According to the results obtained previously by other authors [10], it confers bioadhesive properties to the systems where it was incorporated (microspheres) due to its high hydrophilicity.

During this work starch was modified with 2-isocyanatoethyl methacrylate and a polymer containing urethane linkages and carbon-carbon double bonds was obtained. This modified starch was crosslinked via UV irradiation using Irgacure 2959 by CIBA $^{\circledR}$ as the photoinitiating agent. The choice of this photoinitiator was based on previous studies that proved this compound to be well tolerated over a wide range of cell types and chemical concentrations [13]. Since the main goal of this work was to develop a controlled drug delivery system for ophthalmic application, was used, two different drugs (timolol maleate and sodium flurbiprofen) were incorporated in the synthesized matrix in order to study the release kinetics.

\section{Experimental procedure}

\subsection{Materials}

Starch (St) and 2-isocyanatoethyl methacrylate (IEMA) were purchased from Sigma-Aldrich Chemical Co. (Spain), and the photoinitiator Irgacure ${ }^{\circledR} 2959$ (Ir2959) was obtained from Ciba Specialty Chemicals Inc. All other chemicals and solvents were of analytical-grade and were used as supplied without further purification.

\subsection{Starch modification and film preparation}

St hydroxyl groups were modified by IEMA through the isocyanate groups present in IEMA, resulting in the formation of urethane bonds. To protect the isocyanate groups, the reaction was performed in dimethyl sulfoxide (DMSO) and in the absence of oxygen [14]. Therefore, a 10\% (w/v) DMSO St dispersion was prepared and IEMA was added to the mixture. The ratio of $\mathrm{OH}: \mathrm{NCO}$ groups used was $3: 2$. The reaction was performed by constant stirring of the reagents in a conventional two-neck round-bottomed glass flask placed in a water bath at $60^{\circ} \mathrm{C}$ for $24 \mathrm{~h}$, under a nitrogen atmosphere. When the reaction time ended, the resulting solution was removed from the water bath and it was kept in the dark at room temperature. The photoinitiator (Ir2959) was then added in a percentage of $4 \%(\mathrm{w} / \mathrm{w})$ of IEMA, and stirred until complete solubilization. The resultant solution was irradiated for the period of 2 min by using a UV lamp (Model UVGL-48, Multiband UV, from Mineral light ${ }^{\circledR}$ Lamp). After this period of time, a film was obtained as a result of St-IEMA UV curing. This film (St-IEMA-UV) was then washed with distilled water for $24 \mathrm{~h}$ and finally dried in a vacuum oven at room temperature until constant weight. The dry film was used for the subsequent studies.

\subsection{Attenuated total reflectance-Fourier transform infrared (ATR-FTIR)}

The modified starch (St-IEMA) as well as cured film (StIEMA-UV) were analyzed by attenuated total reflectance-Fourier transform infrared (ATR-FTIR) on a Magma-IR ${ }^{\text {TM }}$ Spectrometer 750 (Nicolet Instrument Corp.), equipped with a Golden Gate Single Reflection Diamond ATR. Spectra were recorded on an average of 32 scans with a resolution of $4 \mathrm{~cm}^{-1}$ and in the frequency range of $400-4000 \mathrm{~cm}^{-1}$.

\subsection{Water sorption capacity and water contact angle determination}

The water sorption capacity of the St-IEMA-UV film was determined by gravimetry. The dried samples were weighted $\left(W_{0}\right)$ and immersed in artificial lachrymal fluid (in $\mathrm{g} / \mathrm{L}: \mathrm{NaCl} 6.7, \mathrm{KCl} 1.0$, $\mathrm{NaHCO}_{3} 2.0, \mathrm{CaCl}_{2}$ 0.08, pH 7.4 [15]) at either room temperature or at $37^{\circ} \mathrm{C}$. The immersed polymers were removed at predetermined time intervals, the water remaining on their surface was gently wiped and the polymers were weighed until the equilibrium state was reached. The sorption capacity was calculated by using Eq. (1):

Sorption capacity $=\frac{W_{t}-W_{0}}{W_{0}} \times 100$

where $W_{\mathrm{t}}$ and $W_{0}$ are the weight of the samples at time $t$ in the artificial lachrymal fluid and the initial (dry) weight of the sample, respectively. The experiment was carried out in triplicate. Data are expressed as mean \pm standard error of the mean (S.E.M.).

During this work, water contact angle (WCA) measurements on the surfaces were performed both for native St and St-IEMA-UV films. The average contact angle for each film were evaluated by static contact angle $(\theta)$ measurements and calculated with a YoungLaplace fit, using an OCA 20 from Dataphysics. The WCA referred in this work was determined as the first stable contact of the drop with the film's surface. All the tests were performed on the air-facing surfaces of the samples. Five measurements on different points were performed to calculate the mean static contact angle $\theta$ and its standard deviation.

\subsection{In vitro biodegradation}

Biodegradation of the St-IEMA-UV film was gravimetrically quantified. It was performed in artificial lachrymal fluid, supplemented with $2 \mathrm{mg} / \mathrm{mL}$ lysozyme [16], at $37^{\circ} \mathrm{C}$ for the period of 6 weeks. This incubation medium was chosen in order to simulate as closely as possible the medium that the films would be subjected to in vivo. The lachrymal fluid contains a large variety of proteins from which lysozyme is one of the primary proteins (reviewed by Johnson and Murphy [17]). Its normal concentrations are between 0.6 and $2.6 \mathrm{mg} / \mathrm{mL}$, representing around $20-40 \%$ of the total tear protein (reviewed by Ohashi et al. [18]). The dried film samples were weighted and placed in the incubation medium. This was refreshed twice a week. At predefined periods of time samples were removed, carefully wiped, dried in a vacuum oven at room temperature and finally weighted ( $n=3$ for each time point). The mass loss was calculated as expressed in Eq. (2):

Mass loss $(\%)=\left(1-\frac{W_{t}}{W_{0}}\right) \times 100$

where $W_{\mathrm{t}}$ and $W_{0}$ are the dry sample weight at time $t$ and the initial weight of the sample, respectively. Data are expressed as mean \pm standard error of the mean.

One of the removed samples at the 4-week incubation period was held in reserve for SEM analysis.

\subsection{Scanning electron microscopy (SEM)}

In order to visualize the morphology of the prepared films, they were placed over carbon ribbon in an appropriated support and then covered with a copper thin layer. The analyses were performed in a Scanning Microscope JSM-5310 from Jeol with an accelerating voltage of $25 \mathrm{kV}$, and different magnifications were used to observe the surfaces and the cross sections of the membranes. 


\subsection{Mercury intrusion porosimetry}

The pore size distribution and total pore volume of the StIEMA-UV film was determined by mercury intrusion porosimetry (Micromeritics, Model Poresizer 9320). The pore diameter was calculated according to the Washborn equation (Eq. (3)):

$D=\frac{-4 \gamma \cos \theta}{P}$

where $P$ is the applied external pressure to intrude mercury, a nonwetting liquid, into the pores with diameter $D, \gamma$ is the surface tension of mercury and $\theta$ the contact angle between mercury and polymer [19].

The pressures applied in the low pressure and high-pressure domains were from 0.5 to $30,000 \mathrm{psi}$, respectively. These pressures correspond to pore sizes ranging from $360 \mu \mathrm{m}$ and $6 \mathrm{~nm}$. The contact angle of mercury with the St-IEMA-UV film was $140^{\circ}$ and the surface tension of mercury was $485 \mathrm{mN} / \mathrm{m}$.

\subsection{Drug loading into St-IEMA-UV membrane}

To perform the drug delivery studies, the $1 \mathrm{~mm}$ thick St-IEMAUV membrane was cut into $9 \mathrm{~mm}$ diameter discs using a cork borer. These discs were vacuum dried at room temperature and used for the following study. Drug loading was performed by a soaking procedure, as described elsewhere [20,21]. In short, the samples were immersed either in $5 \mathrm{~mL}$ of timolol maleate or sodium flurbiprofen aqueous solutions $(0.5 \mathrm{mg} / \mathrm{mL})$ for 3 days at $37^{\circ} \mathrm{C}$. The equilibrium drugs concentrations in the outer solutions were measured by UVVIS spectrophotometry at their maximum absorvance: $294 \mathrm{~nm}$ for timolol and $247 \mathrm{~nm}$ for flurbiprofen, using a UV-VIS spectrophotometer (model V550, Jasco). The amount of drugs loaded by each sample was calculated as the difference between the initial and the final concentrations in the surrounding solution. After loading, each sample was quickly rinsed with distilled water and dried in a vacuum oven at room temperature until constant weight. The loading procedure was performed in triplicate for each template drug.

\subsection{In vitro drug release and data treatment}

Each previous loaded polymer disc was put in an individual vial with $5 \mathrm{~mL}$ of $0.9 \% \mathrm{NaCl}$ aqueous solution. The vials were kept at a $37^{\circ} \mathrm{C}$ water bath with gently constant stirring for the whole release process. At predetermined time intervals, $0.5 \mathrm{~mL}$ of the release medium was sampled and replaced with fresh $0.9 \% \mathrm{NaCl}$ solution to imitate the drainage by tears and maintain sink conditions. The drug concentrations present in the samples were determined by UV-VIS spectrophotometry at their maximum absorvance. Moreover, blank polymer discs (i.e., unloaded polymer discs) were also prepared and used as references. For each time point, their residual absorvance was monitored. The difference in the absorvance between the loaded and the blank samples was used to calculate the drug concentration in the release media [22]. Each data point is the average of three cumulative individual measurements and results are expressed as mean \pm standard error of the mean.

The drugs' release kinetics study was based in the proposal of Ritger and Peppas [23] where the quantity of drug release from the polymeric matrix is analyzed according to the Eq. (4):

$\frac{M_{t}}{M_{\infty}}=k t^{n}$

where $M_{t}$ is defined as the mass of released drug at time $t, M_{\infty}$ is the mass of released drug at the equilibrium, $k$ is a kinetics constant and $n$ is the difusional exponent which is indicative of the release kinetics order. In this equation $M_{t} / M_{\infty}$ represents the fractional release of the drug normalized with respect to the equilibrium conditions [24].

Eq. (4) has been frequently used in the literature as it gives information on difusional mechanism. The value $n=0.5$ indicates a Fickian diffusion process and $n=1$ indicates Case II transport in anomalous diffusion. In the representation of $\ln \left(M_{t} / M_{\infty}\right)$ vs. $\ln t$, a linearity must be observed until values of the fraction $M_{t} / M_{\infty} \leq 0.60$ (first $60 \%$ of the release data), and $n$ and $k$ are got from the slope and the intercept, respectively.

In this condition, Fick's diffusion is characterized by $t^{1 / 2}-$ time dependence of the solute release [25]. Fick's second law may be written as

$\frac{M_{t}}{M_{\infty}}=4\left(\frac{D}{\pi \ell^{2}}\right)^{1 / 2} t^{1 / 2}$

where $k=4\left(\mathrm{D} / \pi l^{2}\right)^{1 / 2}, D$ is the diffusion coefficient and $l$ is the polymer thickness.

\section{Results and discussion}

\subsection{Synthesis and ATR-FTIR analysis}

A photocrosslinkable polymer based on starch was synthesized by reacting part of its hydroxyl groups with 2-isocyanatoethyl methacrylate. This reaction resulted in the formation of urethane groups and allowed the insertion of terminal carbon-carbon double bonds in starch structure.

The formation of the urethane groups was monitored by ATRFTIR by detection of the bands at $3361 \mathrm{~cm}^{-1}(\mathrm{~N}-\mathrm{H}$ hydrogen bonded stretching) and at $1560 \mathrm{~cm}^{-1}(\mathrm{C}-\mathrm{N}$ stretching and $\mathrm{N}-\mathrm{H}$ bending from urethane group) (Fig. 1).

Fig. 1 shows that the band correspondent to the isocyanate groups present in the IEMA spectrum at $2260 \mathrm{~cm}^{-1}$, was no longer detected at the one of the St-IEMA, meaning that all the isocyanate groups had reacted with the starch $\mathrm{OH}$ groups.

The presence of the carbon-carbon double bonds in the modified starch was also confirmed by visualization of the bands at $1676 \mathrm{~cm}^{-1}$ and $822 \mathrm{~cm}^{-1}$.

The modified starch was then photocrosslinked by UV irradiation using Ir2959 as a photoinitiator. The scheme representing the chemical reactions involved throughout the synthesis process is presented in Fig. 2.

An ATR-FTIR analysis was also performed to the photocrosslinked polymer. The resultant spectrum is presented in Fig. 3.

The main difference registered between the ATR-FTIR spectra performed before and after UV irradiation is the decrease in the intensity of one of the bands correspondent to the carbon-carbon double bonds $\left(1676 \mathrm{~cm}^{-1}\right)$ and total disappearance of the other $\left(822 \mathrm{~cm}^{-1}\right)$. These factors suggest that although the crosslinking reaction was extensive after $2 \mathrm{~min}$ of irradiation, some double bonds were still present in the polymer structure.

\subsection{Water sorption capacity and water contact angles}

The swelling capacity of a polymer is a determinant parameter when they are designed for biomedical applications and bioadhesive systems are no exception. The swelling behaviour of systems based on mucoadhesive polymers has a significant influence on several of their properties, like adhesiveness, stability and release of incorporated drugs [26]. The water uptake ability of hydrophilic polymers is partially responsible for their strong adhesion to the mucosa. This fact is based on the contribution of several effects like absorption, swelling and capillary effects that lead to a water flux from the underlying tissue to the polymer [27]. 


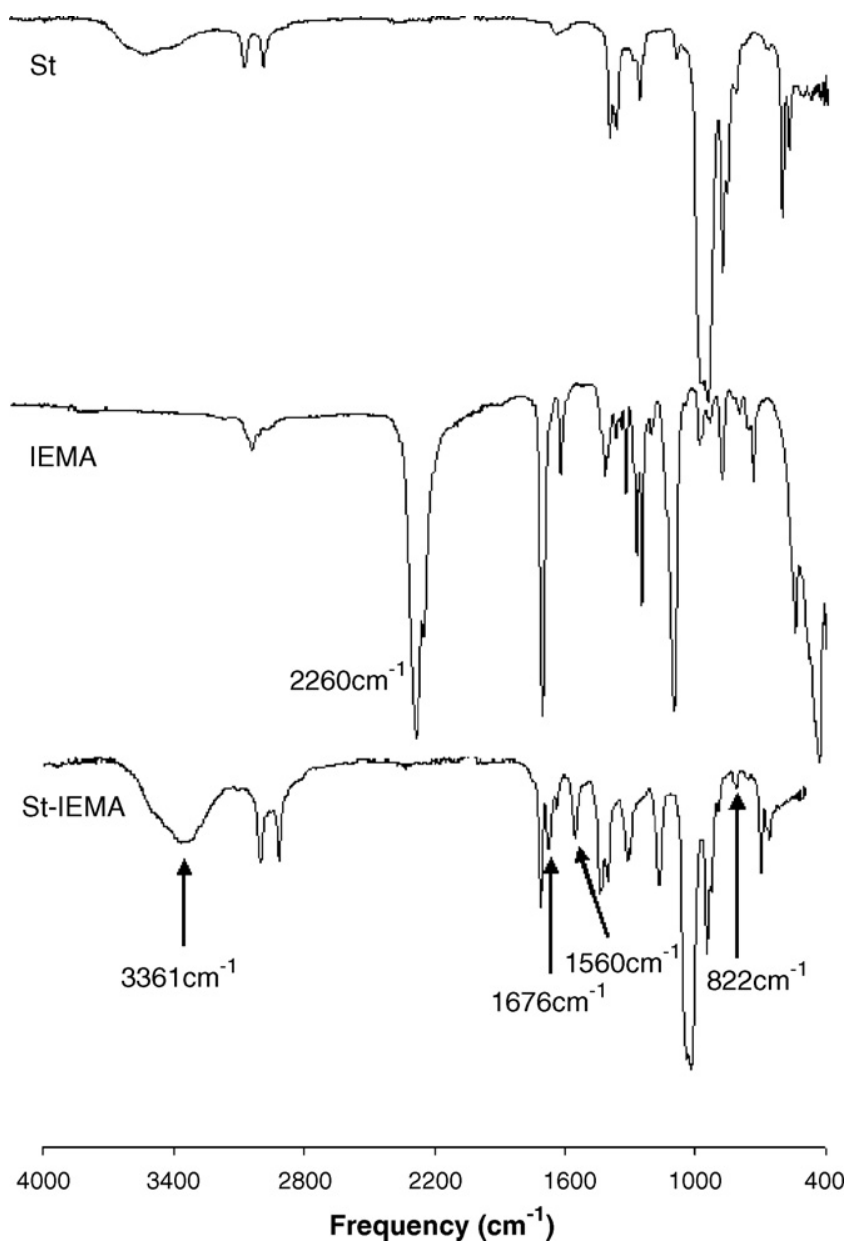

Fig. 1. ATR-FTIR spectrum obtained for starch (St) and modified starch (St-IEMA) solutions in DMSO $(10 \% \mathrm{~m} / \mathrm{v})$ as well as for 2-isocyanato-ethyl methacrylate (IEMA).

The swelling capacity of the synthesized films was evaluated at two different temperature values (room temperature and $37^{\circ} \mathrm{C}$ ) in artificial lachrymal fluid. Both assays suggested that the crosslinked polymeric material presented a hydrophilic character, reaching a maximum weight increase of $78.8 \pm 2.2 \%$ at room temperature (approximately $25^{\circ} \mathrm{C}$ ) and of $90.6 \pm 2.1 \%$ at $37^{\circ} \mathrm{C}$ (Fig. 4). The obtained values revealed a statistically significant difference between the two experimental conditions ( $p<0.05$; unpaired twotailed Student's $t$-test).

The polymer may therefore be classified as a hydrogel. This difference between swelling values when measurements are performed at different temperatures was stated by other authors when working with other polysaccharides (chitosan and sodium alginate) based drug delivery systems [28]. The same authors obtained higher swelling values at $37^{\circ} \mathrm{C}$ than at $25^{\circ} \mathrm{C}$. This difference was attributed to the higher kinetic energy of water molecules moving inside the polymer matrix. However, when starch is concerned, the interactions between the chains that are present in its structure should also be considered. It is known that when starch molecules are placed in warm water, their crystalline structure may be disrupted. This phenomenon allows water molecules to link by hydrogen bonding to hydroxyl groups of amylose and amylopectin which become exposed and causes an increase in swelling. Both factors can influence swelling capacity dependence with temperature of starch-based materials.

The polymers hydrophilicity was also confirmed by the measurement of the water contact angles. The WCA for the
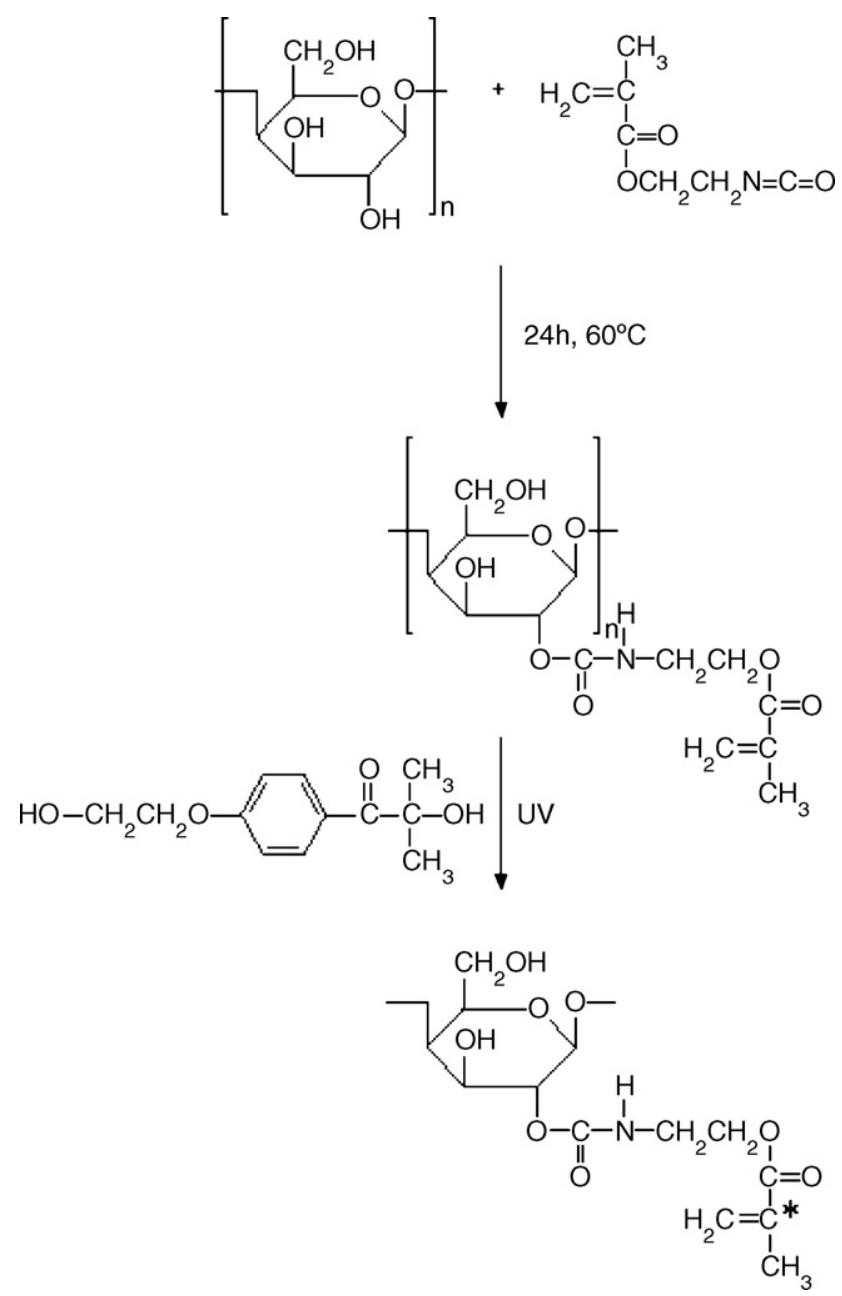

Fig. 2. Schematic representation of the chemical reactions involved in the film preparation. In a first step St is modified with IEMA resulting in the introduction of carbon-carbon double bonds in its structure. After this modification, Ir2959 was added to the solution that was finally subjected to UV irradiation resulting in the formation of a film (St-IEMA-UV).
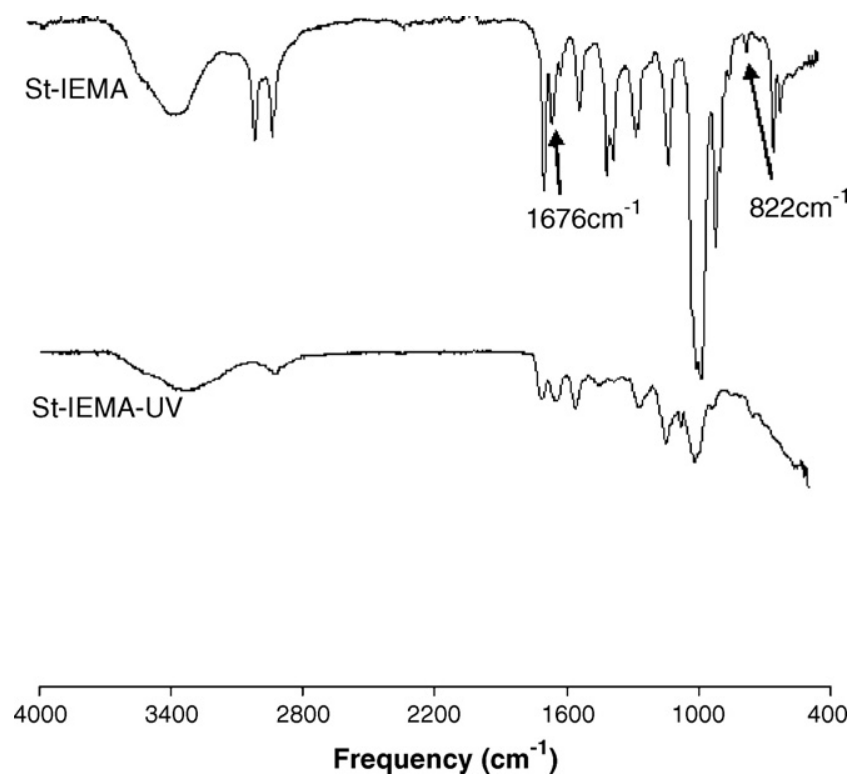

Fig. 3. ATR-FTIR spectrum obtained for the modified starch (St-IEMA) solution (10\% in DMSO) and for the totally dry crosslinked St-IEMA film (St-IEMA-UV) after rinsing with distilled water. 


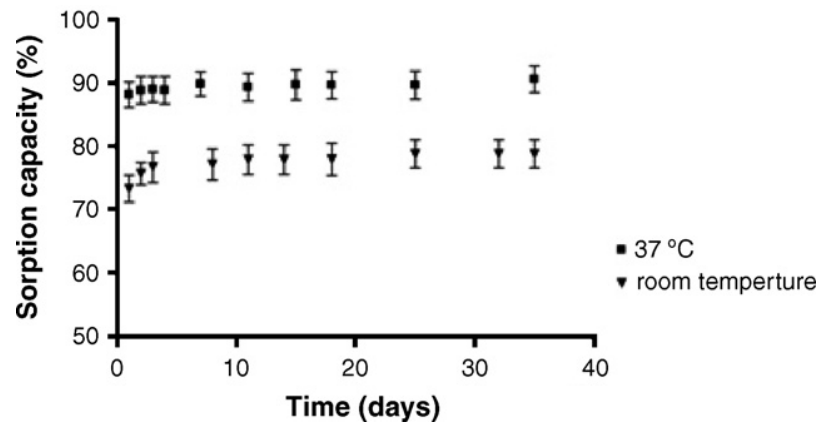

Fig. 4. Swelling capacity of St-IEMA-UV films at two different temperature values: room temperature $(\mathbf{v})$ and $37^{\circ} \mathrm{C}(\boldsymbol{\square})$ in artificial lachrymal fluid. Data are expressed as mean \pm S.E.M. $(n=3)$.

native St film was $59.6^{\circ} \pm 3.6$, while the WCA for the StIEMA-UV film was slightly higher, $71.2^{\circ} \pm 1.5$. This means that even though the hydrophilicity of the modified starch film was decreased by the synthesis method, the polymeric matrix remains hydrophilic. This property is a very important one as previously explained.

\subsection{In vitro biodegradation}

The findings describing the degradation process of polymeric biomaterial are of significant importance in the estimation of its lifetime within the body. Furthermore, it may regulate the release profile of the incorporated drugs.

The degradation of the film was evaluated as mass loss (\%) by gravimetric method (weight measured in the dry state) when incubated in lachrymal fluid supplemented with lysozyme, at $37^{\circ} \mathrm{C}$. According to the obtained results, it was possible to verify that

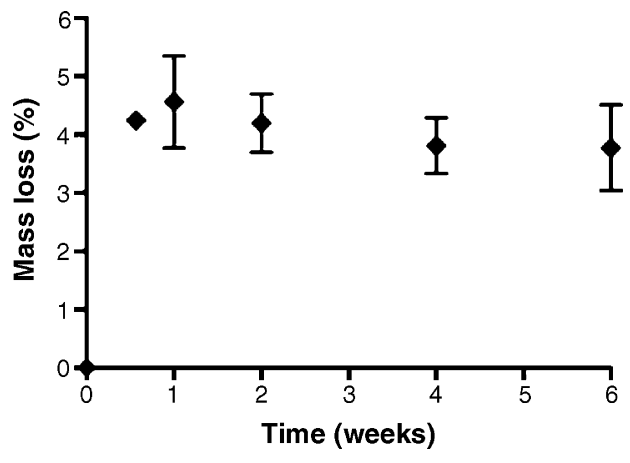

Fig. 5. Mass loss (\%) of the St-IEMA-UV film incubated in artificial lachrymal fluid, supplemented with $2 \mathrm{mg} / \mathrm{mL}$ lyzosime, at $37^{\circ} \mathrm{C}$. Data are expressed as mean \pm S.E.M. $(n=3)$.

biodegradation of the samples remains almost constant during experimentation time (Fig. 5).

In fact, weight loss of the samples was registered as being near $4 \%$ trough the 6 weeks that they were kept in incubation medium. If standard error is considered, there are no significant statistical differences between degradation values along time. This means that biodegradation of the synthesized films occurs almost exclusively during the first $48 \mathrm{~h}$ of incubation.

The possible degradation mechanisms in this system are hydrolysis and enzymatic degradation. Nonetheless, the degradation of the St-IEMA-UV film is proposed to be due to hydrolysis, as the enzyme present in the incubation medium has no specificity for the $\alpha$-D-glucosidic linkages of starch.

The morphology of the surface of the synthesized membrane (St-IEMA-UV) was observed by SEM (Fig. 6A and B) at different magnifications. Using SEM, it is possible to visualize morphologic
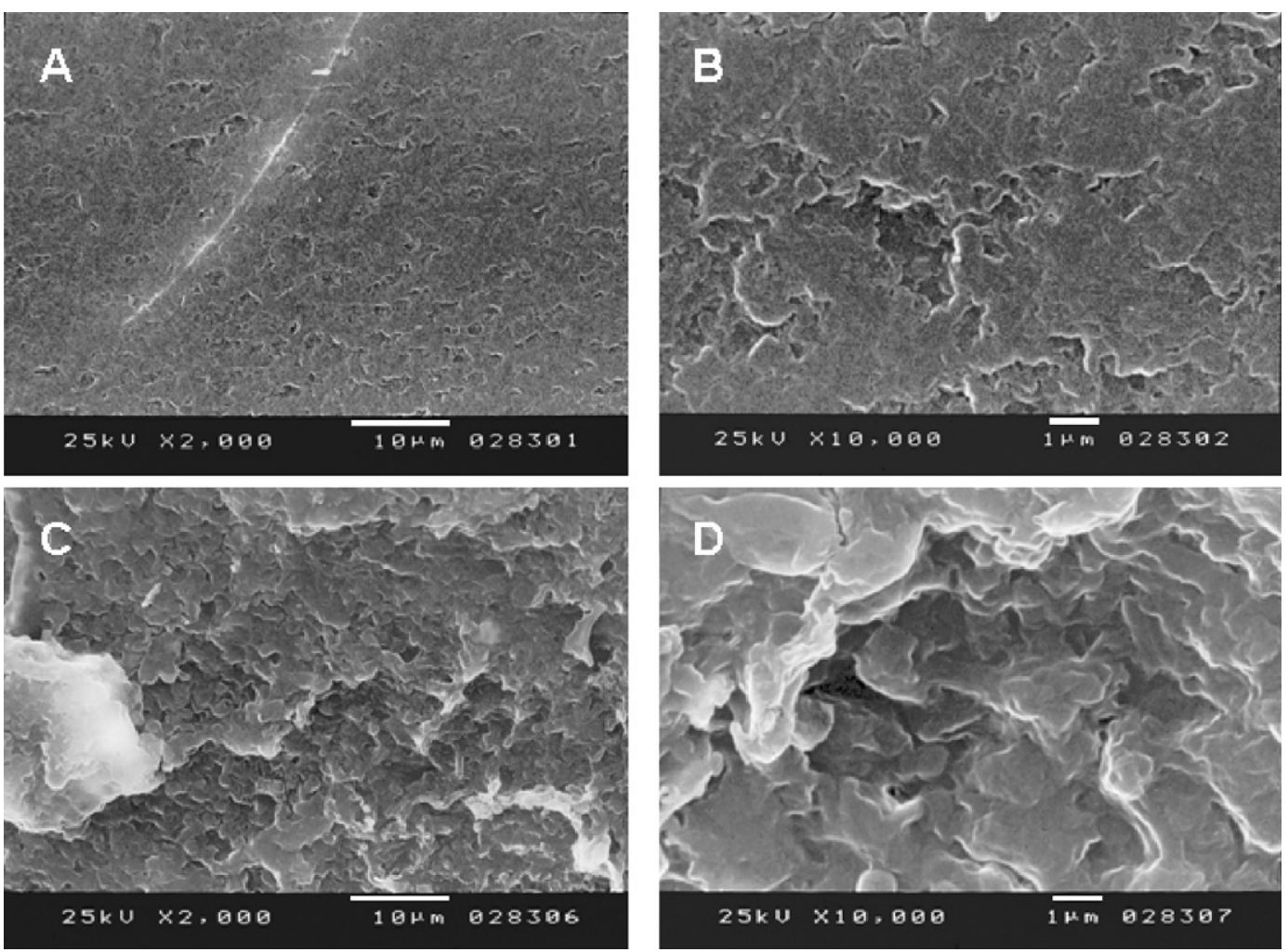

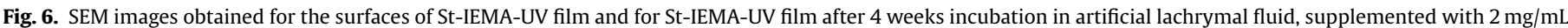
lysozyme. 
details in samples with no accuracy loss. The membrane showed some roughness at the surface with holes and a few cavities. Indeed, this texture gave evidence of the porosity of the material.

This technique allowed also the visualization of the variation in the integrity of the membrane structure after biodegradation. From the analysis of Fig. 6 (C and D) one can clearly see the pores size increasing due to the degradation process. The presence of lysozyme in the incubation medium, did not affect the degradation behaviour. This finding is in accordance with the gravimetric study.

\subsection{Drug loading capacity}

St-IEMA-UV membranes were loaded with timolol and flurbiprofen at $37^{\circ} \mathrm{C}$. Their loading capacity is represented in Fig. 7. It was found that the amount of different loaded drugs is very similar, around $4.7 \mathrm{mg} / \mathrm{mg}$ dry polymer. This observation suggested a comparable number of loading sites within the matrix, possibly due to the interactions capability with drugs and conformational features of the membrane networks.

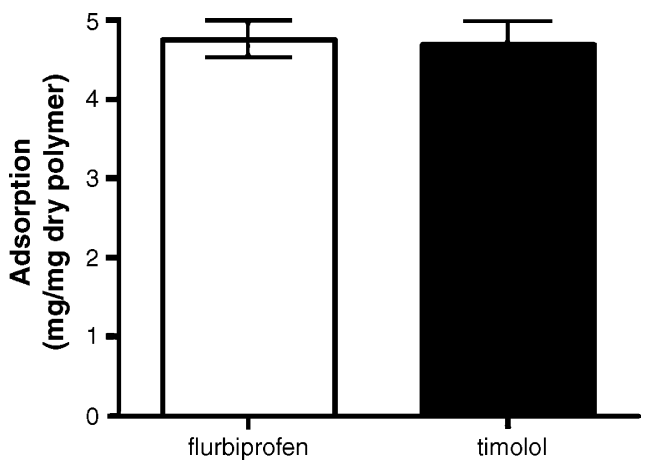

Fig. 7. Drugs adsorption capacity into St-IEMA-UV membrane. Three-day incubation in $0.5 \mathrm{mg} / \mathrm{mL}$ flurbiprofen or timolol at $37^{\circ} \mathrm{C}$.

Morphological analysis was also performed on the loaded and unloaded films by scanning electronic microscopy (Fig. 8). SEM images showed an evident topologic modification of the film membrane surface after the drugs incorporation. The superficial
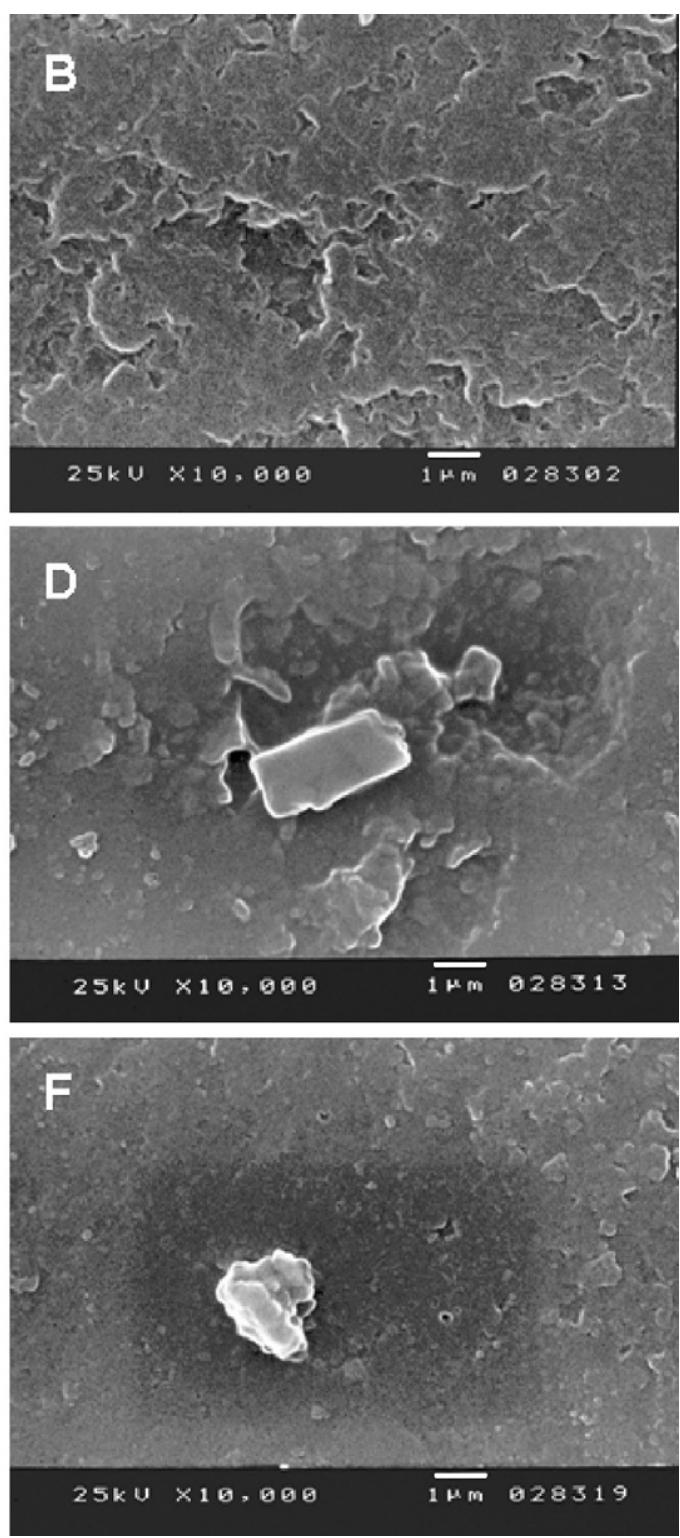

Fig. 8. Surface SEM images of St-IEMA-UV membrane without drug loading (A-B), and loaded with timolol (C-D) and flurbiprofen (E-F). 


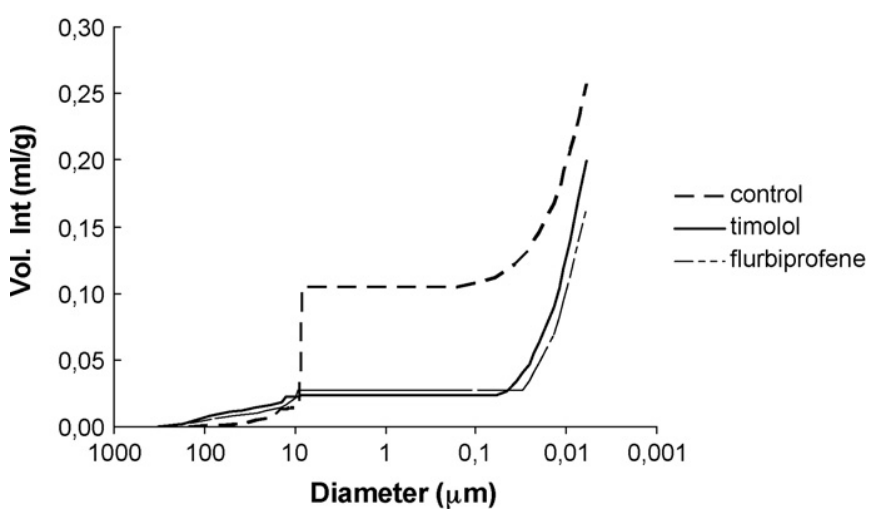

Fig. 9. Mercury intrusion porosimetry. Pore size cumulative volume distribution curve of the St-IEMA-UV membrane without any treatment (control) or drug loaded.

roughness deformation was perfectly visible, not only in size but also in the amount of holes. The morphology was alike for both drugs. Films' surface became smoother and with less porosity in comparison to the unloaded film (Fig. 8A and B). Furthermore, some small granular-shaped particles could also be seen. However, the timolol loaded membrane surface (Fig. 8C and D) seemed to have bigger holes in relation to the flurbiprofen loaded one (Fig. 8E and F).

In addition to the topography study, the porosity of the material was analyzed by mercury intrusion porosimetry. Porosity is a very important factor that controls the swelling drug release behaviour. These features depend upon the penetration of the aqueous medium into the pores of the material. The pore size distribution is presented in Fig. 9. The St-IEMA-UV film exhibited most of its pores sized about $10 \mu \mathrm{m}$ of diameter.

The material showed $33.81 \%$ of total porosity, whereas loaded samples demonstrated to have a considerably lower porosity. In fact, the porosity observed was $24.12 \%$ and $20.86 \%$ for timolol and flurbiprofen loaded membranes, respectively. Despite the reduction of total pore volume, the drugs incorporation into the crosslinked matrix caused an increase of small pore size volume as well. These quantitative findings were consistent with the qualitative SEM observation.

\subsection{Release profiles of drugs}

It was our purpose to study the behaviour of our drug release system in a simulated ocular environment. In this way, the loaded samples were placed in release medium imitating lachrymal fluid $\left(0.9 \% \mathrm{NaCl}\right.$ solution) at $37^{\circ} \mathrm{C}$. The normalized fractional release of drugs plotted versus of time is shown in Fig. 10. It could be seen that both drugs obtained a similar steady-state velocity. However, the difference in the initial drugs release kinetics must be stressed. Flurbiprofen revealed to have faster release kinetics than timolol. According to Tuovinen et al. [29] similar timolol release profile was obtained for starch-acetate microparticles. To a first approximation it may be considered that this initial fast release was probably due to the fraction of drugs which were adsorbed or weakly bond to the surface area of the membrane .

The drugs diffusion processes were studied according to Eq. (4) for the first $60 \%$ drug release data. It was observed that timolol release was a Case II diffusion with $n$ value close to 1 ( $n=0.96$; $r^{2}=0.98$ ). In the words of Alvarez-Lorenzo et al. [21] the release profile of timolol from crosslinked hydroxyethyl methacrylate (HEMA) hydrogels was found to be slow with a $n$ value characteristic of a non-Fickian, anomalous transport. These authors ascribed this finding to a possible increase in hydrogen bonds with the hydroxyl

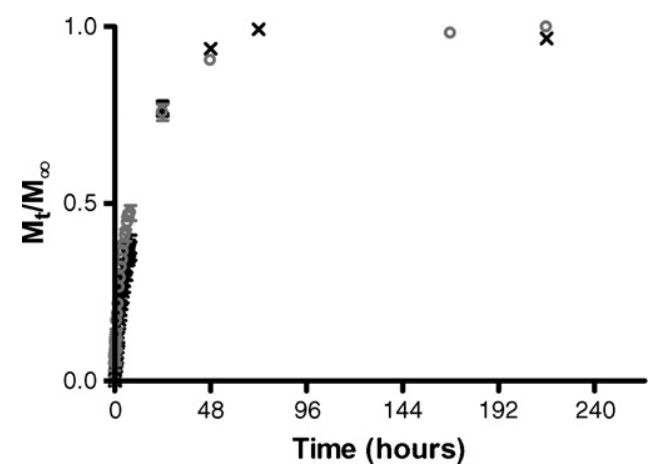

Fig. 10. Release profiles of timolol $(x)$ and flubiprofen $(\bigcirc)$ in $0.9 \% \mathrm{NaCl}$ solution at $37^{\circ} \mathrm{C}$ from loaded St-IEMA-UV membranes. Results are expressed as mean \pm S.E.M. $(n=3)$.

and/or amino groups of timolol. On the other hand, the diffusion process of flurbiprofen from St-IEMA-UV film gave evidence for a Fickian diffusion process with a $n$ value of $0.56\left(r^{2}=0.98\right)$.

The drug diffusion through the polymeric matrix seems to be the rate-limiting step. Indeed, from the degradation studies one can take for granted that the matrix degradation is negligible and can be out of conjecture. The diffusion coefficients found confirm the delayed release of timolol $\left(2.38 \times 10^{-9} \mathrm{~cm}^{2} / \mathrm{s}\right)$ in comparison to flurbiprofen $\left(15.34 \times 10^{-9} \mathrm{~cm}^{2} / \mathrm{s}\right)$.

Nevertheless, this polymeric system showed a sustained release profile for both template drugs for several weeks and seems useful for therapeutical system.

\section{Conclusion}

The proposed photocrosslinked starch-based polymer with urethane linkages has demonstrated to be a biocompatible and biodegradable hydrogel. The differences observed were related only to the degradation profiles. In what morphology is concerned, St-IEMA-UV polymer demonstrated a rough and porous morphology. Best possible conditions were obtained for drug loading and release. It was possible to verify that the drugs' incorporation into the polymer matrix was mainly controlled by the swelling behaviour of the polymer, rather than the different characteristics of each drug. In addition, the drug loading led to a pore size decrease of the polymeric network, as well as smoother surface morphology. Drug release studies proved to have different difusional behaviours. Timolol revealed to be a Case II difusional anomalous process, whereas flurbiprofen diffusion was a typical Fickian release pattern. However, diffusion of both drugs from the polymeric matrices seems to be the main driving force of the release pattern.

Despite the differences in the release profiles of the drugs tested, the polymeric material synthesized in this work may therefore be considered a promising biomaterial for use in ophthalmic applications as sustained drug delivery system.

\section{References}

[1] E. Mannermaa, K.S. Vellonen, A. Urtti, Adv. Drug Deliv. Rev. 58(2006) 1136-1163.

[2] K. Jarvinen, T. Jarvinen, A. Urtti, Adv. Drug. Deliv. Rev. 16 (1995) 3-19.

[3] Y. Ali, K. Lehmussaari, Adv. Drug Deliv. Rev. 58 (2006) 1258-1268.

[4] J.L. Greaves, C.G. Wilson, Adv. Drug. Deliv. Rev. 11 (1993) 349-383.

[5] J.C. Lang, Adv. Drug Deliv. 16 (1995) 39-43.

[6] B.A. Booth, L.V. Denham, S. Bouhanik, J.T. Jacob, J.M. Hill, Drugs Aging 24 (2007) 581-602 (22).

[7] A. Ludwig, Adv. Drug Deliv. Rev. 57 (2005) 1595-1639.

[8] J.K. Vasir, K. Tambwekar, S. Garg, Int. J. Pharm. 255 (2003) 13-32.

[9] A. Bernkop-Schnürch, Drug Discov. Today: Technol. 2 (2005) 83-87.

[10] G. Fundueanu, M. Constantin, A. Dalpiaz, F. Bortolotti, R. Cortesi, P. Ascenzi, E. Menegatti, Biomaterials 25 (2004) 159-170. 
[11] J. Mulhbacher, P. Ispas-Szabo, M. Ouellet, S. Alex, M.A. Mateescu, Int. J. Biol. Macromol. 40 (2006) 9-14.

[12] J.D. Smart, Adv. Drug Deliv. Rev. 57 (2005) 1556-1568.

[13] C.G. Williams, A.N. Malika, T.K. Kima, P.N. Mansonb, J.H. Elisseef, Biomaterials 26 (2005) 1211-1218

[14] K.-J. Shon, S.-T. Lim, B. Yoo, Starch/Stärke 57 (2005) 363-369.

[15] A. Rozier, C. Mazuel, J. Grove, B. Plazonnet, Int. J. Pharm. 57 (1989) 163-168.

[16] V. Rebeix, F. Sommer, B. Marchin, D. Baude, T.M. Duc, Biomaterials 21 (2000) 1197-1205

[17] M.E. Johnson, P.J. Murphy, Prog. Retinl. Eye Res. 23 (2004) 449-474.

[18] Y. Ohashi, D. Murat, K. Tsubota, Clin. Chim. Acta 369 (2006) 17-28.

[19] E.W. Washburn, Proc. Natl. Acad. Sci. 7 (1921) 115-116.

[20] H. Hiratani, C. Alvarez-Lorenzo, J. Control. Release 83 (2002) 223-230.
[21] C. Alvarez-Lorenzo, H. Hiratani, J.L. Gómez-Amoza, R. Martínez-Pacheco, C Souto, A. Concheiro, J. Pharm. Sci. 91 (2002) 2182-2192.

[22] D. Gulsen, C.-C. Li, A. Chauhan, Curr. Eye Res. 30 (2005) 1073-1080.

[23] P.L. Ritger, N.A. Peppas, J. Control. Release 5 (1987) 23-36.

[24] C.S. Brazel, N.A. Peppas, Eur. J. Pharm. Biopharm. 49 (2000) 47-58.

[25] N.A. Peppas, L. Brannon-Peppas, J. Food Eng. 22 (1994) 189-210.

[26] S.A. Mortazavi, J.D. Smart, J. Control. Rel. 25 (1993) 197-203.

[27] D. Guggi, M.K. Marschütz, A. Bernkop-Schnürch, Int. J. Pharm. 274 (2004) 97-105.

[28] A. El-Kamel, M. Sokar, V. Naggar, G.S. Al, AAPS PharmSci. 4 (2002), article 44.

[29] L. Tuovinen, S. Peltonen, M. Liikola, M. Hotakainen, M. Lahtela-Kakkonen, A. Poso, K. Järvinen, Biomaterials 25 (2004) 4355-4362. 